Mathematical topology

\title{
Solving a knotty problem
}

\section{from Ian Stewart}

Occasionally scientific discoveries are made simultaneously by several different people. In topology, an extreme case occurred recently, and is described as follows in the Bulletin of the American Mathematical Society. "The editors received, virtually within a period of a few days in late September and early October 1984 , four research announcements, each describing the same result - the existence and properties of a new polynomial invariant for knots and links. There was variation in the approaches taken by the four groups and variation in corollaries and elaboration. These were: $A$ new invariant for knots and links by Peter Freyd and David Yetter; A polynomial invariant of knots and links by Jim Hoste; Topological invariants of knots and links by W.B.R. Lickorish and Kenneth C. Millett; and A polynomial invariant for knots: a combinatorial and an algebraic approach by A. Ocneanu. It was evident from the circumstances that the four groups arrived at their results completely independently of each other, although all were inspired by the work of [Vaughan] Jones . . . The degree of simultaneity was such that, by common consent, it was unproductive to try to assess priority. Indeed ... there is enough credit for all to share in."

As a compromise, all six authors combined together to write a paper (Bull. Am. Math. Soc. 12, 239; 1985) stating their common main result and explaining each group's particular viewpoint. Curiously, there are considerable differences in the proof and the point of view adopted. The new invariant is simple and powerful, and it is surprising that it has eluded topologists for so long.

To a topologist a knot is any simple closed curve embedded in threedimensional space. Two knots are (topologically) equivalent if there is a continuous transformation of the surrounding three - dimensional space that carries one knot to the other. Similarly, a link is a finite set of simple closed curves, with an analogous definition of equivalence. The main problem is to decide whether two

$$
\begin{aligned}
& \text { (O } P_{L}=y z^{-1}+x^{-1} y^{2} z^{-1}-x^{-1} z, \\
& \text { (1) } P_{L}=x^{-2} z^{2}-2 x^{-1} y-x^{-2} y^{2} \\
& \text { (1) } P_{L}=y^{-2} z^{2}-2 x y^{-1}-x^{-2} y^{-1} z^{2}-x y^{-1}-x^{-1} y-1 .
\end{aligned}
$$

given knots or links are equivalent, and this is generally approached by using the idea of a topological invariant. This comprises some mathematical data (usually of a combinatorial or algebraic character) associated with each knot in such a way that equivalent knots have the same data. Thus knots with different data cannot be equivalent but knots with the same data need not always be equivalent.

The first effective invariant was found by Hans Reidemeister in the 1920 s, and it enabled him to give the first rigorous proof that knots exist. His invariant can be described in terms of the knot diagram, which is just a picture of the knot in which all crossings are neatly separated from each other and with a small gap drawn in the lower strand of each crossing, much as a road map shows a bridge. Pretend that this gap is real - the knot then splits up into several components; at each crossing one component forms the overpass while
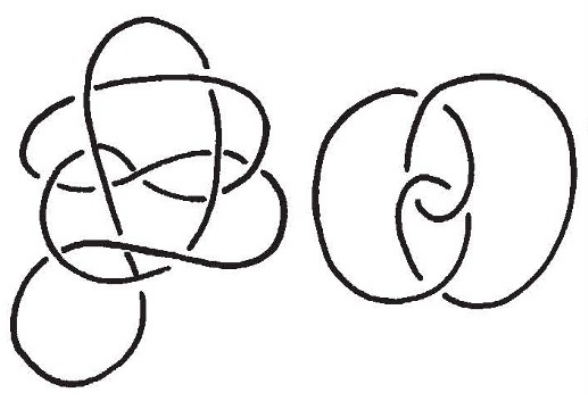

two others form the underpass. Say that the knot can be $n$-coloured if it is possible to assign to each component a number $0,1, \ldots, n-1$ in such a way that: (1) at least two components have different numbers; and (2) at each crossing the sum of the numbers on the two underpass components is equal to twice the number on the overpass, up to a multiple of $n$.

Then, by analysing how deformations of the knot alter its diagram, and thus affect colourability, it can be shown that the set of integers $n$ for which a given knot is $n$-colourable is an invariant. An ordinary overhand knot is 3-colourable but not 5-colourable; a 'figure of eight' knot is 5-colourable but not 3-colourable; and an unknotted loop is not $n$-colourable for any values of $n$. Hence all three are different.

A more sophisticated invariant, the Alexander polynomial, associates with each knot or link $K$ a polynomial in one variable $t$. For the trefoil it is $t^{2}-t+1$, and for the figure eight $t^{2}-3 t+1$. Again the invariant is good enough to distinguish the two. John Conway (Computational Problems in Abstract Algebra 379; Pergamon, Oxford, 1970) developed a method for computing Alexander polynomials based on a relation between the Alexander polynomials of a given link $K$ and two modifications of it, $L$ and $M . L$ is obtained from $K$ by taking a single crossing and changing the overpass to an underpass; $M$ is obtained by cutting both strands at the crossing and joining them in pairs so that they do not cross at all. Vaughan Jones (Bull. Am. Math. Soc. 12, 103; 1985) applied the theory of Van Neumann algebras
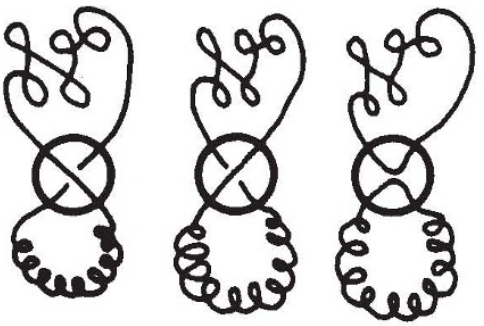

- a topic in functional analysis - to derive a new knot polynomial, also in one variable, satisfying a very similar relation.

The latest invariant can be considered either as a homogeneous polynomial in three variables or as an inhomogeneous polynomial in two. In terms of three variables it is the unique such function $P$ on (equivalence classes of) knots such that:

(1) $x P_{K}(x, y, z)+y P_{L}(x, y, z)=0$

(2) $P_{K}(x, y, z)=1$

when $K$ is an unknotted circle. Relation (1) is inspired by a property of Alexander and Jones polynomials, and both of these can be expressed in terms of $P$. But the new invariant has stronger properties. For example, for a left-handed trefoil knot it is $x^{-2} z^{2}-2 x^{-1} y-x^{-2} y^{2}$, whereas for a righthanded trefoil it is $y^{-2} z^{2}-2 x y^{-1}-x^{2} y^{-2}$. Hence, the trefoil and its mirror image are different. A reef knot and a granny knot also have different invariants $P$, and hence are inequivalent. Both of these are known results, but until now they have had very difficult proofs and cannot be obtained from the Alexander or Jones polynomials.

The proof of the existence of the invariant $P$ is technical but relatively straightforward given the property of relation (1). It may be accomplished in several ways. Freyd and Yetter use a ringtheoretical argument involving braid groups. Hoste uses geometrical and combinatorial methods. Lickorish and Millett also use braids as well as some of Conway's ideas about tangles. Ocneanu's approach is similar to Jones's, involving number theory and functional analysis.

The hard part is to recognize the importance of relation (1) to begin with: the idea is almost too simple to be credible. The surprise is that it really does work, and beautifully. Some ideas can be discovered at almost any time, but lie fallow for years until the moment is ripe: this would appear to be an extreme case. And mathematicians can take heart from this discovery - not every new idea need be more complicated than old ones.

Ian Stewart is in the Mathematics Institute, University of Warwick, Coventry CV4 7AL, UK. 